OPEN ACCESS

Edited by:

Qiwei Zhang,

Southern Medical University, China

Reviewed by:

Youichi Suzuki,

Osaka Medical College, Japan

Takashi Irie,

Hiroshima University, Japan

${ }^{*}$ Correspondence:

Bin Liu

kjkliubin@126.com

Pujun Gao

gpj0411@163.com

Wei Wei

wwei6@jlu.edu.cn

Specialty section:

This article was submitted to

Virology,

a section of the journa

Frontiers in Microbiology

Received: 05 July 2018 Accepted: 20 September 2018

Published: 08 October 2018

Citation:

Wang $D$, Guo $H$, Chang J, Wang D, Liu B, Gao P and Wei W (2018) Andrographolide Prevents

EV-D68 Replication by Inhibiting the Acidification of Virus-Containing

Endocytic Vesicles.

Front. Microbiol. 9:2407.

doi: 10.3389/fmicb.2018.02407

\section{Andrographolide Prevents EV-D68 Replication by Inhibiting the Acidification of Virus-Containing Endocytic Vesicles}

\author{
Dongyin Wang ${ }^{1,2}$, Haoran Guo ${ }^{2}$, Junliang Chang ${ }^{2,3}$, Dong Wang ${ }^{1,2}$, Bin Liu ${ }^{4 *}$, Pujun Gao ${ }^{1 *}$ \\ and Wei Wei ${ }^{2 *}$
}

'Department of Hepatology, The First Hospital of Jilin University, Jilin University, Changchun, China, ${ }^{2}$ Institute of Virology and AIDS Research, The First Hospital of Jilin University, Changchun, China, ${ }^{3}$ Changchun Institute of Biological Products, Changchun, China, ${ }^{4}$ Department of Hand Surgery, The First Hospital of Jilin University, Changchun, China

Enterovirus D68 (EV-D68) has emerged as a significant respiratory pathogen that can cause severe respiratory disease and acute neurologic disease. At present, there are no approved antiviral agents or vaccines for EV-D68. In this study, we demonstrate that andrographolide (ADO), an active component of Andrographis paniculata, exerts substantial antiviral activity against EV-D68 infection. ADO treatment dramatically inhibited EV-D68 RNA replication $\left(E_{50}=3.45 \mu \mathrm{M}\right)$ and protein synthesis without producing significant cytotoxicity at virucidal concentrations. ADO-treated cells did not show any changes in host immune activation, EV-D68 attachment, or viral $5^{\prime}$ UTR activity. Using a $\mathrm{pH}$-sensitive fluorescent indicator system for endocytosis in living cells, we found that ADO prevented the acidification of endocytic vesicles after receptor-mediated endocytosis. Finally, we showed that ADO inhibited the viral replication of circulating isolated EV-D68 strains. In summary, our results demonstrate that $\mathrm{ADO}$ suppresses EV-D68 replication by targeting the maturation of virus-containing endosomes of EV-D68. This mechanism represents a promising strategy for drug development.

Keywords: enterovirus D68, andrographolide, endocytosis, acidification, drug development

\section{INTRODUCTION}

The genus Enterovirus (EV) of the family Picornaviridae includes several medically important pathogens such as poliovirus, echoviruses, coxsackieviruses, numbered enteroviruses, and rhinoviruses (Baggen et al., 2018). In past decades, several enteroviruses have emerged as serious public health concerns. Recently, enterovirus D68 (EV-D68; originally classified as human rhinovirus 87) (Ishiko et al., 2002), caused a large outbreak of severe lower respiratory tract disease in North America. EV-D68 was first isolated in California from patients with pneumonia and bronchiolitis in 1962 (Schieble et al., 1967) and has since been infrequently reported in association with a wide range of clinical manifestations including mild-to-severe respiratory disease (Kaida et al., 2011; Huang et al., 2017). In 2014, the largest outbreak of EV-D68 infection occurred in the United States (Midgley et al., 2014, 2015). Most patients were children with severe respiratory conditions that necessitated intensive care. Patients with a history (Baggen et al., 2018) of asthma or reactive airway disease were more likely to require ventilator support (Midgley et al., 2015). Since the 2014 outbreak, an increasing number of clusters of EV-D68 infection have been reported 
in Europe, Asia, and Oceania (Messacar et al., 2015, 2016; Oermann et al., 2015; Reiche et al., 2015; Wei et al., 2016). EV-D68 has also been implicated in polio-like neurological disorders such as acute flaccid myelitis (Greninger et al., 2015; Maloney et al., 2015).

EV-D68 differs from other enteroviruses in that it is most often detected in cases of respiratory illness and has biological similarity to rhinovirus (Blomqvist et al., 2002; Oberste et al., 2004; Mertz et al., 2015). EV-D68 is a small, icosahedral virus with a single, positive-stranded RNA genome. The viral genome contains only 1 open reading frame (ORF) that encodes viral polyprotein, which is self-digested by viral proteins $2 \mathrm{~A}$ and $3 \mathrm{C}$ to yield four structural proteins (VP1, VP2, VP3, and VP4) and seven non-structural proteins $(2 \mathrm{~A}, 2 \mathrm{~B}, 2 \mathrm{C}, 3 \mathrm{~A}, 3 \mathrm{~B}, 3 \mathrm{C}$, and 3D) (Baggen et al., 2018). The viral crystal structure of EV-D68 also shows similarity to that of human rhinovirus (Oberste et al., 2004). The EV-D68 capsid consists of 60 copies of each of the subunit proteins VP1, VP2, VP3, and VP4 and has a deep surface depression (canyon) circling each of the 12 pentameric vertices, which is thought to be responsible for viral receptor recognition (Liu et al., 2015b). Previous studies have suggested that cell surface sialic acid is required for host cell infection by EV-D68 (Liu et al., 2015a; Baggen et al., 2016). We recently identified neuro-specific intercellular adhesion molecule 5 (ICAM-5/telencephalin) as a functional entry receptor for sialic acid-dependent and -independent EV-D68 viruses (Wei et al., 2016).

Despite the spread of EV-D68 viruses, there are no effective vaccines or antiviral agents available for clinical use. It was recently reported that three antiviral drugs in clinical trials for enteroviral infections (pleconaril, pocapavir, and vapendavir) failed to inhibit EV-D68 infection (Liu et al., 2015b; Rhoden et al., 2015; Sun et al., 2015).

Andrographis paniculata Nees has been traditionally used for centuries in Asia to treat various diseases such as respiratory infection, fever, diarrhea, and bacterial dysentery (Panraksa et al., 2017). Andrographolide (ADO) is a bicyclic diterpenoid lactone and is thought to be the major bioactive component of A. paniculata, exerting anti-inflammatory, anti-cancer, and antiviral activities (Wintachai et al., 2015; Panraksa et al., 2017). Previous studies have shown that ADO has activity against several viruses including the human immunodeficiency virus (Calabrese et al., 2000), hepatitis B virus (Chen et al., 2014), hepatitis C virus (Lee et al., 2014), dengue virus (Panraksa et al., 2017), chikungunya virus (Wintachai et al., 2015), and herpes simplex virus (Aromdee et al., 2011). In this study, we evaluated the activity of ADO against the EV-D68 virus. Our results reveal the antiviral activity of ADO and shed light on its utility for the treatment of EV-D68 infections.

\section{MATERIALS AND METHODS}

\section{Cells and Viruses}

Human rhabdomyosarcoma RD cells (ATCC, CCL-136) were cultured in Dulbecco's modified Eagle medium (DMEM; Hyclone $^{\mathrm{TM}}$, cat no: SH30022.01) supplemented with $10 \%$ fetal bovine serum (Biological Industries, REF: 04-001-1) and 1\% penicillin/streptomycin solution. EV-D68 prototype Fermon (ATCC, VR-1826) and EV-D68 circulating strains from the 2014 United States outbreak, US/MO/14-18947 (ATCC, VR-1823D) and US/KY/14-18953 (ATCC, VR-1825D), were propagated in RD cells. A mixture of cell and supernatant was collected at approximately 5 days post-infection and subjected to multiple cycles of freezing and thawing. Then, the mixture was clarified by low-speed centrifugation and passed through a $0.22-\mathrm{mm}$ filter, and viral particles were pelleted through a $20 \%$ sucrose cushion in a SW28 rotor by centrifugation at 28,000 rpm for $90 \mathrm{~min}$. Purified virions were stored at $-80^{\circ} \mathrm{C}$ until use.

\section{Cell Viability Assay}

$\mathrm{RD}$ cells were cultured in 96-well plates with complete medium until they reached $90 \%$ confluence. Then, cell culture medium was removed and replaced with complete medium containing $0.4 \%$ dimethyl sulfoxide (DMSO) or various concentrations of ADO (MCE, HY-N0191) (0, 1.25, 2.5, 5, 10, 20, and $40 \mu \mathrm{M})$. Negative control (only media) and positive control wells $(0.4 \%$ DMSO) were included on each plate. The cells were incubated for $24 \mathrm{~h}$ and then $30 \mu \mathrm{l}$ MTS was added to each well. Three hours later, the color intensity of each well was detected at $490 \mathrm{~nm}$ using a 550 Bio-Rad plate reader. Each data point represents the average of three replicates in cell culture.

\section{Viral Attachment Assays}

For virus attachment experiments, the cells were first washed with cold DMEM, and then EV-D68 viruses were added to the cells. After incubation at $4^{\circ} \mathrm{C}$ for $2 \mathrm{~h}$, treated cells were washed with cold DMEM to remove unbound viruses. Total RNAs were extracted using an RNeasy Mini Kit (Qiagen). The bound virus RNA was determined by using qRT-PCR.

\section{Virus Titer Assay}

Virus titers were determined by endpoint dilution assay (EPDA). Briefly, RD cells were cultured under standard conditions in 96well plates at a density of 10,000 cells per well. EV-D68 was serially diluted (10-fold) with DMEM containing 1\% FBS and added to cells. Virus titers were determined by the appearance of cytopathic effects (CPEs) in RD cells using a microtitration analysis in accordance with the Reed-Muench method (Reed and Muench, 1938).

\section{RNA Quantitation by qRT-PCR}

Total RNA from cells was isolated using Trizol (Life Technologies) according to the manufacturer's instructions, including the DNase I digestion step. Samples were incubated in $10 \mu \mathrm{l}$ of diethyl pyrocarbonate (DEPC)-treated water with $1 \mathrm{x}$ RQ1 RNase-Free DNase buffer, $1 \mu$ l of RQ1 RNase-free DNase (Promega), and $4 \mathrm{U}$ of RNase inhibitor (New England Biolabs) for $30 \mathrm{~min}$ at $37^{\circ} \mathrm{C}$. The DNase activity was inactivated by the addition of $1 \mu \mathrm{l}$ RQ1 DNase stop solution and incubation at $65^{\circ} \mathrm{C}$ for $10 \mathrm{~min}$. The RNA was reverse-transcribed by using random primers and Multiscribe reverse transcriptase from the HighCapacity cDNA Archive Kit (Applied Biosystems, Carlsbad, CA, 
United States), according to the manufacturer's instructions. The cDNA was either used undiluted or serially diluted in DEPC-treated water before the real-time PCR reaction to ensure that the amplification was within the linear range of detection. The StepOne Real-Time PCR system (Applied Biosystems) was used for qRT-PCR amplification. The reactions were performed under the following conditions: $50^{\circ} \mathrm{C}$ for $2 \mathrm{~min}$ and $95^{\circ} \mathrm{C}$ for $10 \mathrm{~min}$, followed by $40 \mathrm{cycles}$ of $95^{\circ} \mathrm{C}$ for $15 \mathrm{~s}$ and $60^{\circ} \mathrm{C}$ for $1 \mathrm{~min}$, followed by a dissociation protocol. Single peaks in the melting curve analysis indicated specific amplicons. qRT-PCR was performed with the following primer sequence: GAPDH forward primer 5'-GCAAATTCCATGGCACCGT-3'; GAPDH reverse primer 5'-TCGCCCCACTTGATTTTGG-3'; EV-D68 forward primer 5'-TGTTCCCACGGTTGAAAACAA-3'; and -EV-D68 reverse primer 5'-TGTCTAGCGTCTCATGGTTTTCAC$3^{\prime}$. IFN- $\beta$ : $5^{\prime}$-TTGTGCTTCTCCACTACAGC-3' (forward) and $5^{\prime}$-CTGTAAGTCTGTTAATGAAG-3' (reverse); ISG56: $5^{\prime}$-CAACCAAGCAAATGTGAGGA-3' (forward) and $5^{\prime}$ AGGGGAAGCAAAGAAAATGG-3' (reverse). The relative levels of EV-D68 RNA in different samples were determined using a comparative $2^{-\triangle \triangle \mathrm{CT}}$ method with normalization against the GAPDH gene (Wei et al., 2016).

\section{Immunoblotting}

Cells were harvested at various time points post-infection, washed twice with cold phosphate-buffered saline (PBS), and lysed in lysis buffer (150 mM Tris [pH 7.5] containing $150 \mathrm{mM}$ $\mathrm{NaCl}, 1 \%$ Triton X-100, and complete protease inhibitor cocktail [Roche]) and loading buffer (0.08 M Tris [pH 6.8] containing $2.0 \%$ SDS, $10 \%$ glycerol, $0.1 \mathrm{M}$ DTT, and $0.2 \%$ bromphenol blue). The solutions were boiled and vortexed for $10 \mathrm{~min}$ and then centrifuged at $14000 \times g$ for $10 \mathrm{~min}$. Supernatant proteins were separated by SDS-PAGE and transferred to nitrocellulose membranes using a semidry apparatus (BioRad). The membranes were probed with primary antibodies (anti-EV-D68 VP1 polyclonal antibody [Genetex, GTX132312] and anti- $\beta$-actin monoclonal antibody [Sigma, A3853]) at $4^{\circ} \mathrm{C}$ overnight, followed by incubation with secondary antibodies (alkaline phosphatase-conjugated goat anti-rabbit IgG [Jackson ImmunoResearch Laboratories, code:115-005-045] and goat antimouse IgG [Jackson ImmunoResearch Laboratories, code: 115055-062]) for $1 \mathrm{~h}$ at $25^{\circ} \mathrm{C}$. The membranes were stained with 5bromo-4-chloro-3-indolyl phosphate and nitrotetrazolium blue chloride (Sigma-Aldrich) and visualized for band quantification.

\section{Immunofluorescence and Confocal Microscopy}

Cells were grown on glass plates (Nest, cat no.: 801001) in the presence of $10 \mu \mathrm{M}$ ADO or $0.1 \%$ DMSO vehicle overnight until they reached subconfluence. EV-D68 was bound to cells for $45 \mathrm{~min}$ at $4^{\circ} \mathrm{C}$ in DMEM containing $1 \%$ FBS. Then, the cells were washed with $\mathrm{PBS}$ and incubated at $37^{\circ} \mathrm{C}$ in $1 \%$ DMEM. At various time points, the cells were fixed with $4 \%$ paraformaldehyde (PFA) for $15 \mathrm{~min}$, permeabilized with $0.3 \%$ Triton X-100 for 5 min, blocked with goat serum (ZSGBBIO, ZLI-9022) for $1 \mathrm{~h} 25^{\circ} \mathrm{C}$, and finally stained with EV-D68
VP1 antibody (1:250) at $4^{\circ} \mathrm{C}$ overnight. The next day, the cells were washed five times with PBS and then incubated with Alexa Fluro ${ }^{\circledR} 488$ conjugated goat anti-rabbit IgG antibody (Life Technologies, A11088) for $1 \mathrm{~h}$ at room temperature. DAPI stain (1:100 dilution, Biotopped, top0221) was added $10 \mathrm{~min}$ prior to visualization under fluorescence microscopy (Olympus, IX5 or Olympus Fluo-View-1000 with a 100X objective lens) with Fluo View software v. 1.7. Representative cells were selected and photographed.

\section{Intraendosomal pH Determination}

We used amine-reactive pHrodo dyes (Life technologies, cat no.: P35368) to detect changes in the $\mathrm{pH}$ of virus-containing endosomes. EV-D68 fermon was purified and dissolved in PBS and incubated with amine-reactive pHrodo dyes for $40 \mathrm{~min}$ at room temperature in the dark before infection. $\mathrm{RD}$ cells were then seeded on glass plates in the presence of $10 \mu \mathrm{M}$ ADO or $0.1 \%$ DMSO vehicle overnight. Then, the cells were infected with dyeconjugated $\mathrm{EV}-\mathrm{D} 68$ virus at $4^{\circ} \mathrm{C}$ for $30 \mathrm{~min}$, washed with $\mathrm{PBS}$ and incubated in $37^{\circ} \mathrm{C}$, and observed at different time points $(1,2$, and $3 \mathrm{~h}$ ) with a confocal microscope.

\section{Luciferase Assay}

To evaluate the effect of ADO on the viral transcriptional activity of EV-D68, we constructed a promoter-driven firefly luciferase plasmid pT7-5'UTR-pGL3 and expression plasmid p3D-VR ${ }_{1012}$. p5'UTR-Luc (200 ng) was transfected or co-transfected with pEV-D68-3D (800 ng) into cells pre-treated with ADO or $0.1 \%$ DMSO vehicle. Luciferase activity was assessed $24 \mathrm{~h}$ after transfection. Briefly, the cells were harvested and lysed with passive lysis buffer and then centrifuged at $12000 \times \mathrm{g}$ for 10 min. Supernatants and luciferase substrate (Promega, E190) were mixed in a 96-well plate and fluorescence was quantified with a Fluoroskan Ascent ${ }^{\mathrm{TM}}$ FL instrument (Thermo Fisher, 5210450).

\section{Statistical Analysis}

All data are shown as the mean value and standard deviation of biological replicates (details are explained in figure legends). Statistically significant differences between groups were estimated using two-tailed unpaired Student's $t$-test with GraphPad Prism (version 6.0) software. $P$-value $<0.05$ was considered statistically significant.

\section{RESULTS}

\section{ADO Inhibits EV-D68 Replication}

Andrographolide is a labdane diterpenoid with broad-spectrum antiviral properties (Figure 1A). To investigate the anti-EVD68 activity of ADO, permissive RD cells were pretreated with $10 \mu \mathrm{M}$ ADO or $0.1 \%$ DMSO vehicle for $16 \mathrm{~h}$, followed by challenge with an EV-D68 prototype Fermon virus (MOI of 0.1 . CPEs were detected at $48 \mathrm{~h}$ post-infection in vehicletreated cells; in contrast, ADO-treated cells showed obvious resistance to CPEs (Figure 1B). qRT-PCR data indicated that ADO treatment decreased viral RNA replication by 98 -fold at 


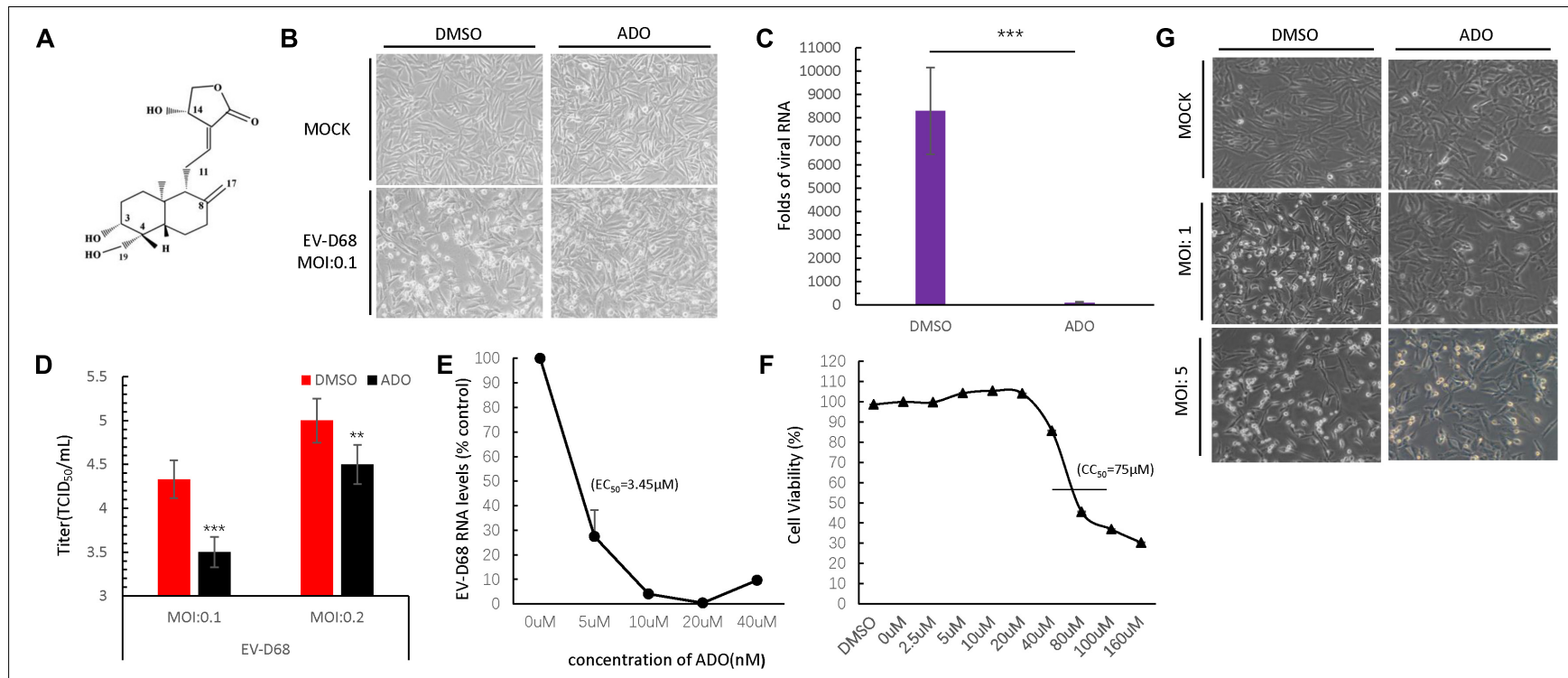

FIGURE 1 | Andrographolide inhibits EV-D68 replication. (A) Structure of andrographolide (ADO; molecular formula: $\left.\mathrm{C}_{20} \mathrm{H}_{30} \mathrm{O}_{5}\right)$. (B) $\mathrm{RD}$ cells were pre-treated with ADO or DMSO vehicle before infection or mock-infection with EV-D68 virus. The cells were incubated under standard conditions. Cytopathic effects (CPEs) were observed 48 h post-infection. (C) qRT-PCR assessment of EV-D68 RNA replication. (D) Determination of progeny viron production. Supernatants were collected $48 \mathrm{~h}$ post-infection and viral titers were determined by standard plaque assay. (E) Effect of ADO on EV-D68 RNA replication (EC $50=\sim 3.45 \mu \mathrm{M})$. (F) Cell viability assay. Cellular toxicity was evaluated by MTS assay and is expressed as the percentage relative to DMSO vehicle-treated control cells. Experiments were performed in triplicate. (G) RD cells were pretreated the same way as cells in (B), then the cells were infected or mock-infected with EV-D68 virus at an MOI of 1 and 5 . CPEs were observed $24 \mathrm{~h}$ post-infection. Error bars indicate the standard deviation ${ }^{*} P<0.05 ;{ }^{* *} P<0.01 ;{ }^{* *} P<0.001$ ).

$48 \mathrm{~h}$ post-infection (Figure 1C). Additionally, ADO treatment significantly decreased titers of EV-D68 progeny virus compared to vehicle treatment (Figure 1D), suggesting that ADO inhibits the proliferation of EV-D68. In addition, we confirmed that ADO exhibited powerful inhibitory effect on EV-D68 at higher MOI of 1 and 5 (Figure 1G). The $\mathrm{EC}_{50}$ value of $\mathrm{ADO}$ against EV-D68 replication was approximately $3.45 \mu \mathrm{M}$ (Figure 1E). We also measured ADO cytotoxicity using the MTS assay and determined that the median lethal concentration of $\mathrm{ADO}$ was $75 \mu \mathrm{M}$ (Figure 1F), which was much higher than the $\mathrm{EC}_{50}$ of antiviral activity.

\section{ADO Inhibits EV-D68 RNA Replication and VP1 Synthesis}

We next sought to determine the part of EV-D68 life cycle that was inhibited by ADO treatment. We analyzed EV-D68 growth curves during infection of RD cells (MOI of 10) in the presence of $10 \mu \mathrm{M}$ ADO or $0.1 \%$ DMSO vehicle (Figure 2A). Viral RNA was quantified using qRT-PCR at 0, 2, 4, 8, 12, and $24 \mathrm{~h}$ post-infection. ADO treatment decreased the amount of intracellular EV-D68 RNA by more than $80 \%$ compared to vehicle at $8 \mathrm{~h}$ post-infection (Figure 2A). At $24 \mathrm{~h}$ postinfection, the amount of EV-D68 RNA in vehicle-treated cells was 19-fold higher than that in ADO-treated cells (Figure 2A). Consistent with this result, there was an observable reduction in EV-D68 VP1 protein expression in ADO-treated cells compared to vehicle-treated cells on immunoblotting (Figures 2B,C) and immunofluorescence assays (Figure 2D). To clarify the dynamics of ADO against EV-D68, a time-of-addition analysis was undertaken, with ADO treatment at 16 and $3 \mathrm{~h}$ prior to infection and 0,2 , and $6 \mathrm{~h}$ post-infection. VP1 expression was evaluated by immunoblotting. The results indicated that ADO treatment post-infection had no obvious effect on EVD68 (Figure 2E). All these data suggested that ADO treatment impeded the early stage of EV-D68 infection.

\section{ADO Does Not Influence EV-D68 3D-5' UTR Activity or Innate Immune Responses}

The $5^{\prime}$ UTR region of enteroviruses is a determinant for viral translation and RNA replication, and the activity of this region can be enhanced by viral polymerase $3 \mathrm{D}$ protein. To assess viral $3 \mathrm{D}$ protein-dependent $5^{\prime}$ UTR activation, we next established a firefly luciferase reporter assay (Figure 3A) and found that ADO treatment had no influence on $5^{\prime}$ UTR activity regardless of 3D expression (Figure 3B).

Previous studies have characterized $\mathrm{ADO}$ as a strong plantderived immunomodulator that affects the NF- $\kappa \mathrm{B}$ and JAKSTAT signaling pathways, which are involved in host innate immune responses (Ding et al., 2017). Therefore, we next measured endogenous mRNA levels of interferon- $\beta$ (IFN- $\beta$ ) and an interferon-stimulated gene ISG56 at $0,2,4,8$, and $12 \mathrm{~h}$ post-infection (Figures 4A,B). IFN- $\beta$ mRNA was increased at $12 \mathrm{~h}$ post-infection, suggesting that EV-D68 infection produced innate immune activation in permissive cells (Figure 4A). ADO treatment at a concentration of $10 \mu \mathrm{M}$ did not have a significant effect on IFN- $\beta$ or ISG56 mRNA expression during EV-D68 infection in RD cells (Figures 4A,B), indicating that ADO did not 
A

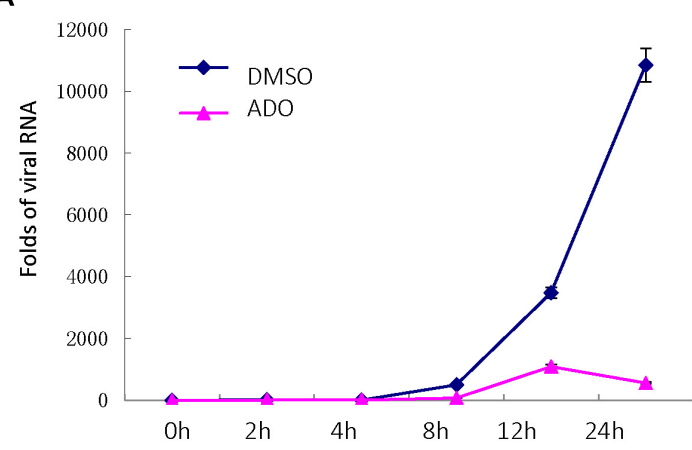

B
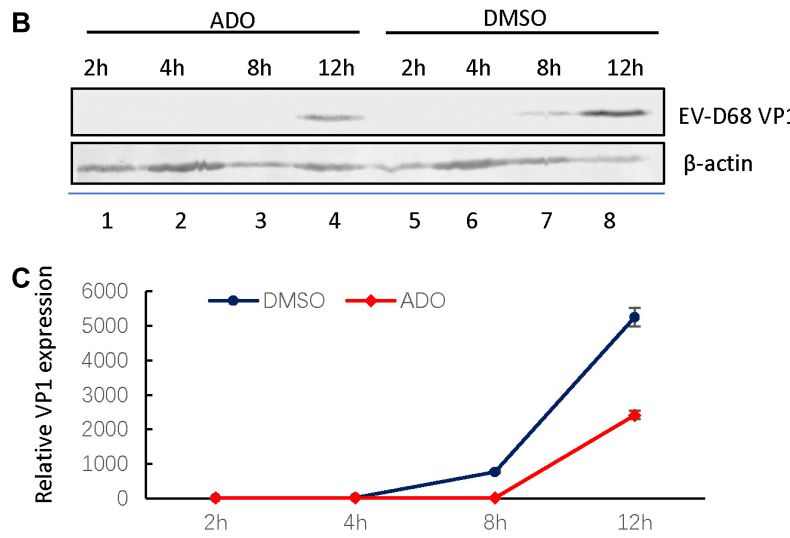

D
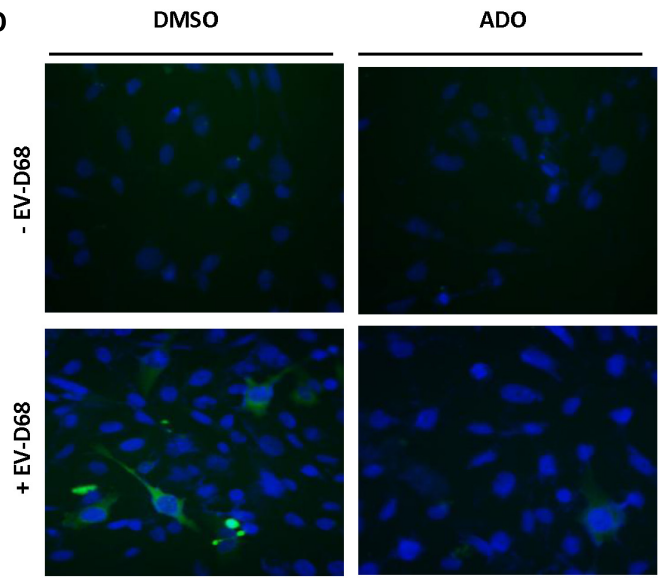

E

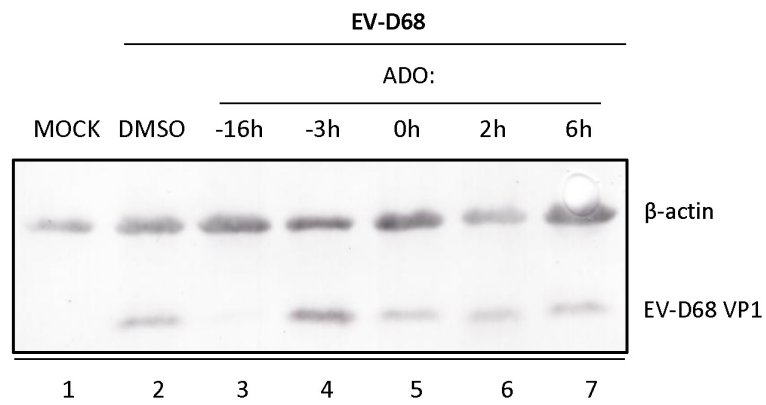

FIGURE 2 | Andrographolide inhibits EV-D68 RNA replication and VP1 synthesis. RD cells were pretreated with andrographolide or DMSO vehicle overnight and subsequently infected with EV-D68. Viral RNA (A) and VP1 (B-D) were measured at several specific time points. (A) Growth curves of EV-D68 in infected cells. (B,C) Immunoblotting of VP1 expression at $8 \mathrm{~h}$ post-infection. (D) Immunofluorescence of VP1 expression at $10 \mathrm{~h}$ post-infection. (E) RD cells were incubated with $10 \mu \mathrm{M}$ $\mathrm{ADO}$ or vehicle only at the indicated time points before and after infection or mock infection with EV-D68 virus. VP1 was analyzed by western blotting $24 \mathrm{~h}$ post-infection, with actin as a control. Experiments were performed in triplicate. Data represent the mean and standard deviation.

A

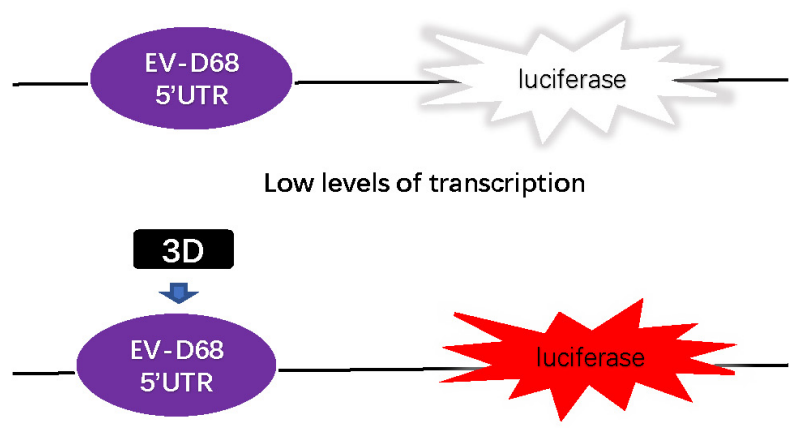

Transcription activated
B

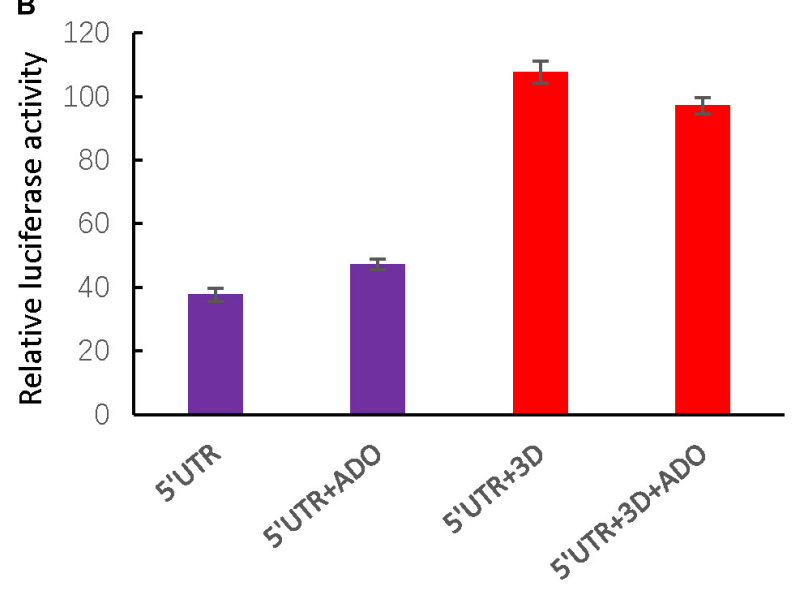

FIGURE 3 | Andrographolide does not influence EV-D68 3D-dependent 5' UTR transcriptional activity or the cellular innate immune response. (A) To assess the effect of andrographolide on EV-D68 transcription, we generated a firefly luciferase reporter assay of $5^{\prime}$ UTR activity. (B) Assay of 3D-dependent 5' UTR activation. p5'UTR-luc (200 ng) was transfected or co-transfected with pEV-D68-3D (800 ng) into HEK293T cells pretreated with ADO or DMSO vehicle in a 12-well plate. Luciferase activity was detected $24 \mathrm{~h}$ post-transfection. 

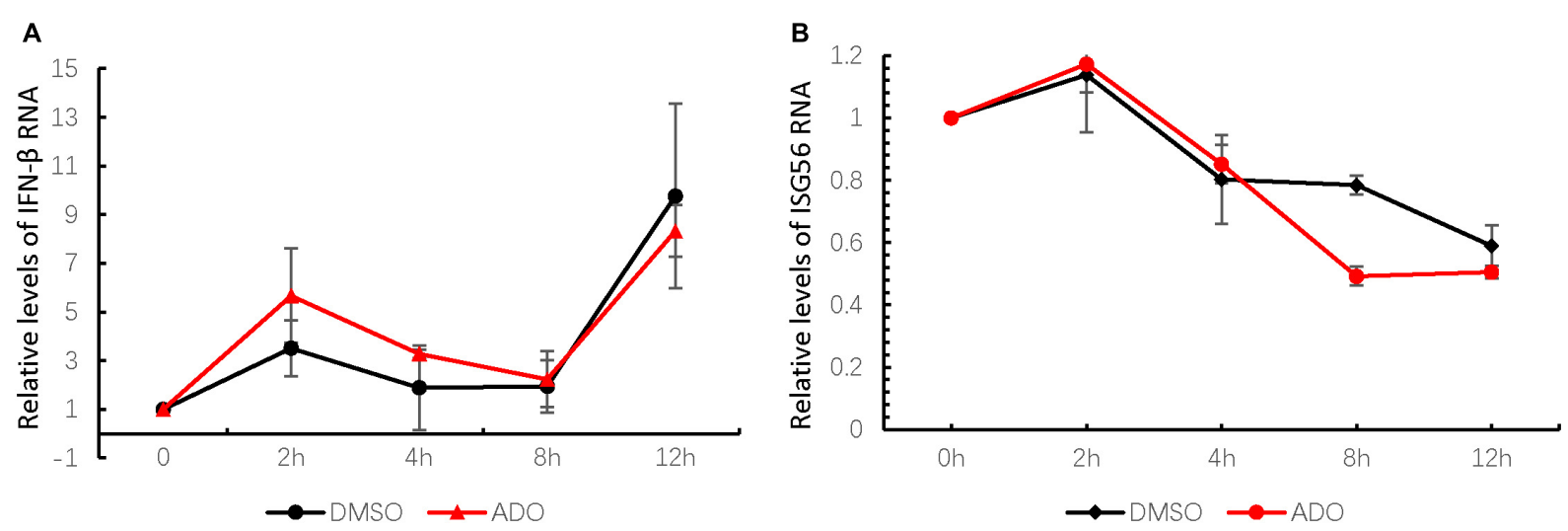

FIGURE 4 | Andrographolide does not alter EV-D68-induced innate immune responses at the cellular level. Gene expression of IFN- $\beta$ (A) and ISG-56 (B), respectively, as indicators of virus-stimulated innate immune response. mRNA was quantified by qRT-PCR at 0, 2, 4, 8, and $12 \mathrm{~h}$ post-infection. Error bars indicate the standard deviation.

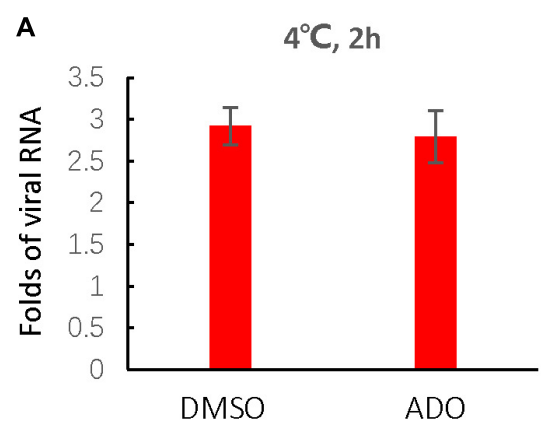

B

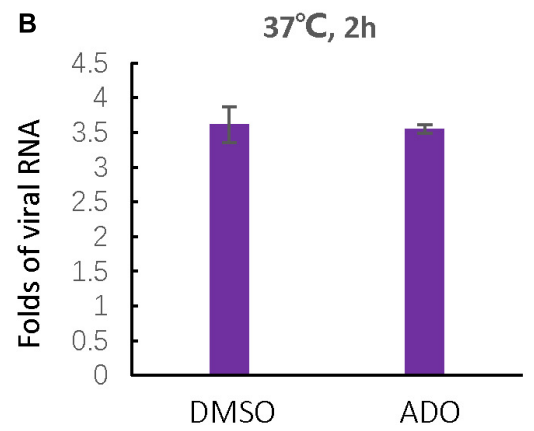

C
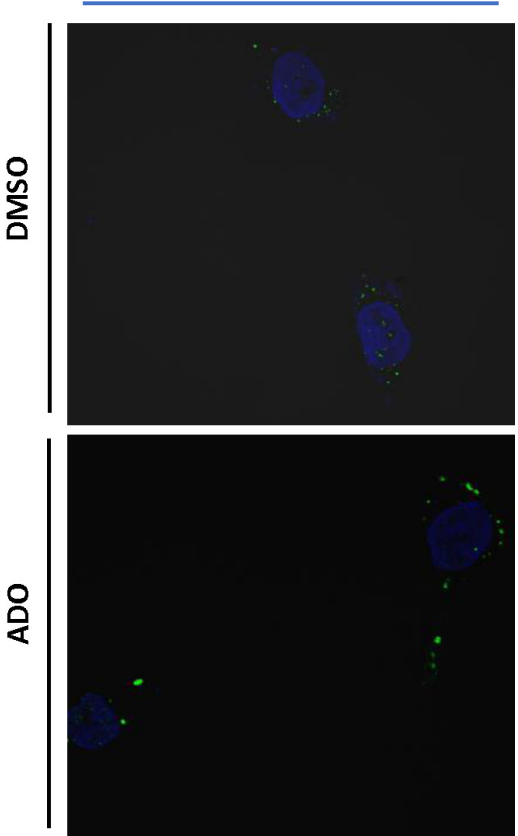

$6 \mathrm{~h}$

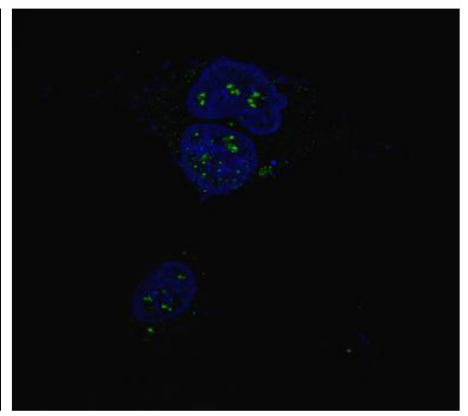

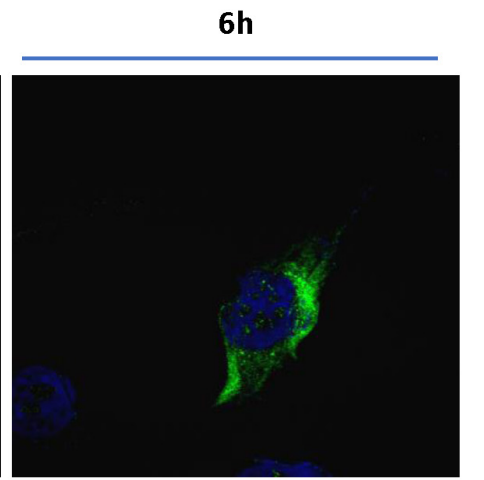

.

FIGURE 5 | Andrographolide inhibits EV-D68 replication in a post-entry step. Andrographolide had no effect on EV-D68 attachment or entry into cells. Cells were pre-treated with ADO or DMSO vehicle in 12-well plates for $16 \mathrm{~h}$ under standard conditions. (A) Cells were incubated at $4^{\circ} \mathrm{C}$ for 30 min before infection and then incubated at $4^{\circ} \mathrm{C}$ for $2 \mathrm{~h}$ to facilitate virus attachment. (B) RD cells were infected with EV-D68 and incubated at $37^{\circ} \mathrm{C}$ for $2 \mathrm{~h}$. qRT-PCR was performed to quantify viral RNA. (C) Assay of intracellular VP1 accumulation. Cells were fixed at 2 and $6 \mathrm{~h}$ post-infection, and VP1 was observed by confocal microscopy. Experiments were performed in triplicate. Error bars indicate the standard deviation.

alter EV-D68-induced innate immune responses at the cellular level.

\section{ADO Does Not Influence EV-D68 Entry Into Cells}

Based on the above studies, we determined that ADO likely inhibited a step in the EV-D68 lifecycle occurring prior to viral RNA replication and protein expression. Therefore, we next investigated whether ADO inhibited EV-D68 replication at the entry step. Viral attachment and entry experiments were performed in the presence of $10 \mu \mathrm{M}$ ADO or $0.1 \%$ DMSO vehicle. Virus attachment and entry were assessed by quantification of intracellular EV-D68 RNA at $2 \mathrm{~h}$ post-infection. Repeated experiments demonstrated that pre-treatment of cells with $\mathrm{ADO}$ had no detectable effect on viral attachment (Figure 5A) or entry (Figure 5B). Next, we detected intracellular 


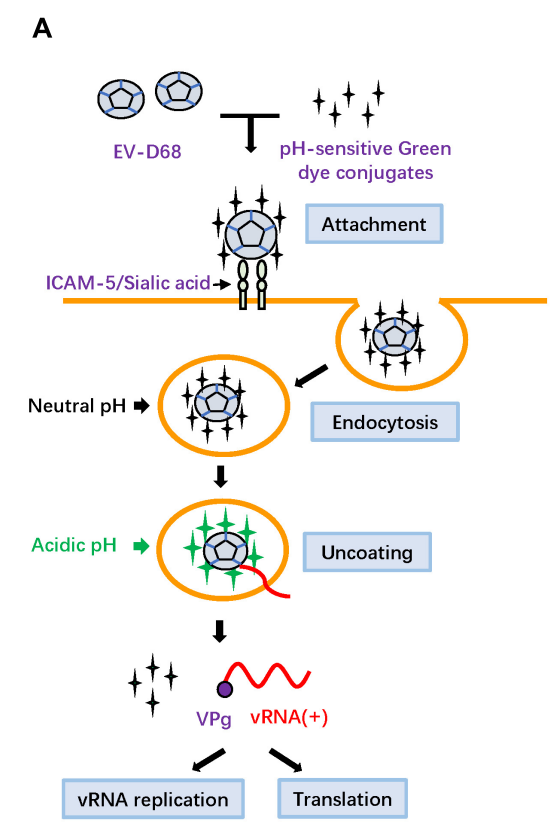

B

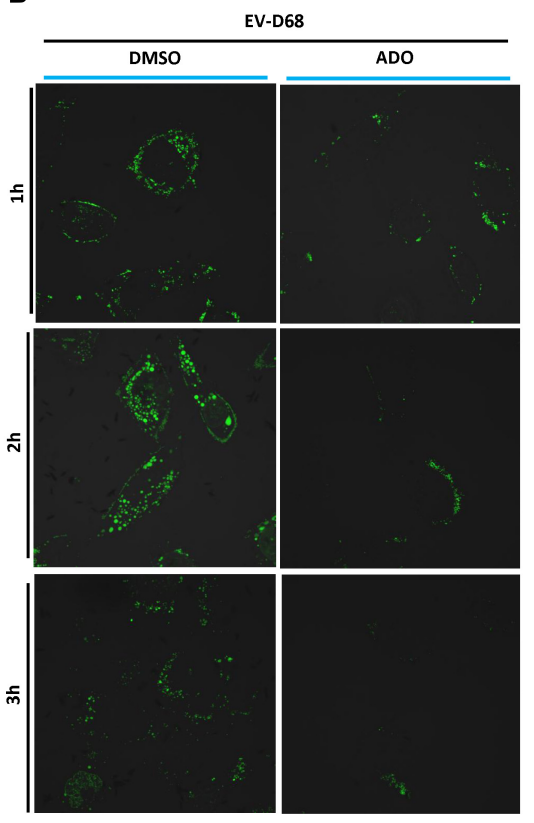

C

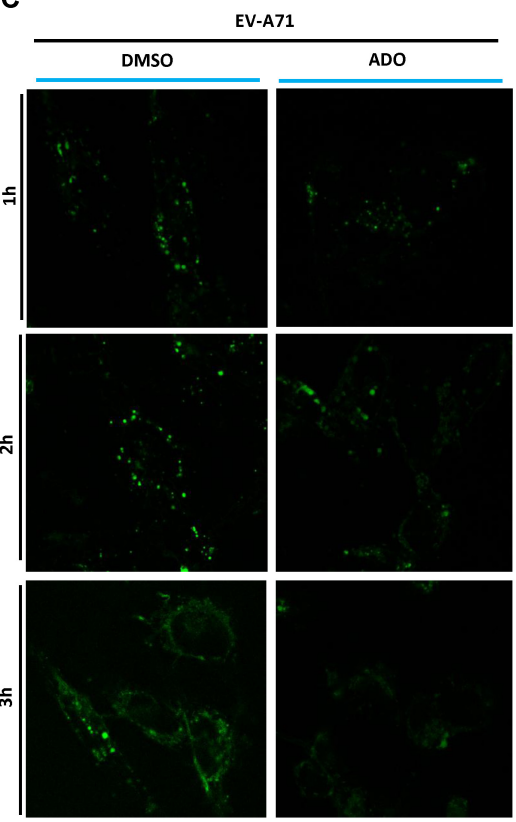

FIGURE 6 | Andrographolide interferes with endosome maturation. (A) Viruses are endocytosed at the cell surface before trafficking toward the nucleus. Gradual acidification occurs alongside endosome maturation. Viral proteins require low pH to undergo conformational changes or protease activation that permit disruption of the endosome membrane and the release of viral particles into the cytoplasm. (B,C) Assay of endosome acidification. EV-D68 and EV-A71 were incubated with $\mathrm{pH}$-sensitive green dye and then infected into pre-treated RD cells. Fluorescence was detected in live cells using Alexa Fluor 488 filters and confocal microscopy at specific time points (1, 2, and $3 \mathrm{~h}$ post-infection). Fluorescence increased with acidity of the surrounding environment.

VP1 protein expression using immunofluorescence at 2 and $6 \mathrm{~h}$ post-infection. Consistent with previous result, there was no effect of $\mathrm{ADO}$ treatment at $2 \mathrm{~h}$ post-infection; however, ADO treatment significantly decreased EV-D68 VP1 protein expression at $6 \mathrm{~h}$ post-infection (Figure 5C). These results collectively demonstrated that ADO inhibited EV-D68 RNA replication and protein accumulation in a post-entry step.

\section{ADO Inhibits the Acidification of EV-D68-Containing Endosomes}

Enteroviruses share a common mechanism in the early steps of viral infection that involves specific receptor binding, viral entry through an endocytic pathway, and subsequent travel through the cytoplasm encased in an endosomal compartment. In most cases, endosomes that contain pathogens undergo maturation to lysosomes to degrade the pathogens. Therefore, the virus must exit the endosome to escape degradation. Viruses use the low $\mathrm{pH}$ of endosomes to activate penetration proteins or undergo conformational changes that facilitate escape (Lagache et al., 2012). To track the process of EV-D68 endocytosis in the presence or absence of ADO treatment, we established a $\mathrm{pH}$-sensitive reporter system during EV-D68 infection by using an acidic $\mathrm{pH}$-sensitive green dye. The dye has a $\mathrm{pH}$ sensitive fluorescence emission that increases in intensity with increasing acidity, with peak fluorescence in the range $\mathrm{pH}$ 5-8 (i.e., the known range of endosome vesicle acidification; Figure 6A). Fluorescence intensity peaked at around $2 \mathrm{~h}$ post-infection and was quickly quenched by $3 \mathrm{~h}$ post-infection in vehicle-treated RD cells (Figure 6B). In contrast, ADOtreated cells exhibited limited fluorescence, suggesting that ADO inhibited the maturation process of EV-D68 endocytosis. Furthermore, we demonstrated that ADO treatment can inhibit the acidification of virus-containing endosome of an enteric enterovirus EV-A71 (Figure 6C). Thus, applying a broadly antiviral activity of ADO against diverse viruses via inhibiting endosome acidification.

\section{ADO Inhibits Isolated Circulating Strains of EV-D68}

Recent studies presented by our lab and others have demonstrated that the EV-D68 prototype virus and circulating strains have different sensitivities for sialic acid-mediated viral replication. Therefore, we examined the antiviral activity of ADO against viral infection by primary EV-D68 isolates (US/MO/14-18947 [MO] and US/KY/14-18953 [KY]). The cells were harvested at $24 \mathrm{~h}$ post-infection for immunoblotting of EV-D68 VP1, and CPEs were observed $48 \mathrm{~h}$ post-infection. The results indicated that $\mathrm{ADO}$ protected $\mathrm{RD}$ cells against both circulating strains of EV-D68 as evidenced by decreases in related CPEs (Figure 7A). Further, ADO treatment significantly decreased intracellular amounts of viral RNA after infection with the MO (Figure 7B) and KY strains (Figure 7C) compared to vehicle treatment. Finally, ADO treatment significantly decreased VP1 protein expression after infection with the MO 

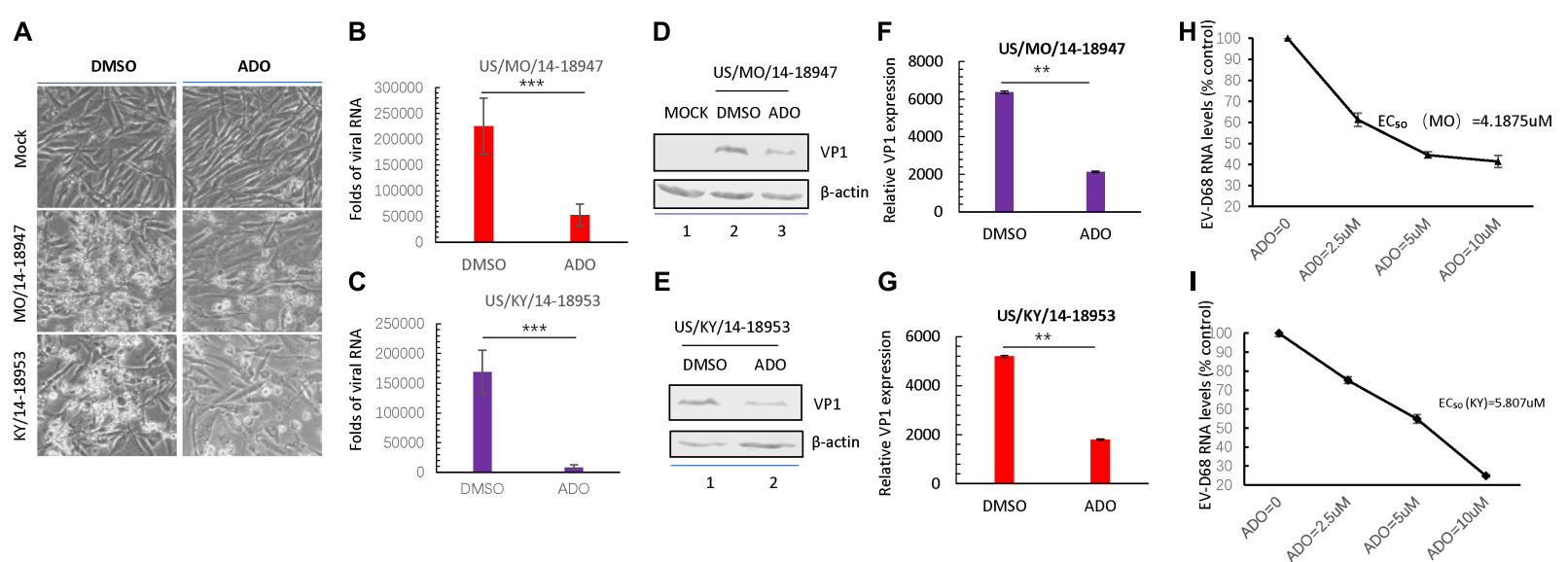

FIGURE 7 | Andrographolide inhibits isolated circulating strains of EV-D68. (A) RD cells were pre-treated with $10 \mu \mathrm{M}$ andrographolide or DMSO vehicle and then infected with US/MO/14-18947 or US/KY/14-18953. Cells were incubated under standard conditions. Cytopathic effects (CPEs) were observed 48 h post-infection. (B,C) qRT-PCR analysis of viral RNA. (D-G) Immunoblotting of VP1 expression at 24 h post-infection with the US/MO/14-18947 (D,F) and US/KY/14-18953 strains (E,G). (H,I) Effect of ADO on RNA replication of EV-D68 circulating strains. US/MO/14-18947:EC $50=\sim 4.1875 \mu \mathrm{M}(\mathbf{H}) ;$ US/KY/14-18953: EC $50=\sim 5.807 \mu \mathrm{M}$. The experiment was performed three times. Error bars indicate the standard deviation $\left.{ }^{*} P<0.05 ;{ }^{* *} P<0.01 ;{ }^{* * *} P<0.001\right)$.

(Figures 7D,F) and KY strains (Figures 7E,G) compared to vehicle treatment. The $\mathrm{EC}_{50}$ values of $\mathrm{ADO}$ against $\mathrm{MO}$ and KY replication were approximately $4.19 \mu \mathrm{M}$ (Figure $7 \mathbf{H}$ ) and $5.807 \mu \mathrm{M}$ (Figure 7I), which was close to that of AOD against EV-D68 prototype Fermon. In summary, ADO exerted broad antiviral effects against both prototype and circulating EV-D68 viruses.

\section{DISCUSSION}

To our knowledge, this is the first study to report the inhibition of viral endocytosis by ADO, a major bioactive constituent of A. paniculata. ADO treatment conferred significant antiviral activity against EV-D68 virus without producing significant toxicity in $\mathrm{RD}$ cells. $\mathrm{ADO}$ treatment was associated with significant decreases in viral RNA replication and protein synthesis. Notably, ADO had no influence on viral attachment, viral endocytosis processes, or innate immune activation in infected cells. By using a $\mathrm{pH}$-sensitive fluorescent dye conjugate to track virus endocytosis in live cells, we revealed that ADO interfered with viral cytoplasmic traffic by preventing vesicle acidification.

Andrographis paniculata has long been an important constituent of traditional herbal medicine in Asia (Kumar et al., 2004) and Scandinavia (Jayakumar et al., 2013), with several studies demonstrating the antiviral, antimicrobial, anti-parasitic, and anti-inflammatory effects of $\mathrm{ADO}$ as a principal active component. Research has demonstrated the therapeutic utility of ADO in respiratory infection by influenza A virus (IAV) by inhibition of IAV-induced RIG-I like receptor signaling pathway in human bronchial epithelial cells (Yu et al., 2014). However, the mechanisms by which ADO exerts broad-spectrum antiviral activity have been poorly defined in previous studies. In our experiments, ADO treatment did not influence EV-D68 infection-mediated innate immune activation as represented by the induction of IFN- $\beta$ and ISG56.

In this study, we analyzed the growth curves of EV-D68 RNA replication and VP1 synthesis during infection and found that ADO dramatically decreased intracellular viral RNA copy numbers and VP1 expression. This finding was consistent with previous studies demonstrating the ability of ADO to prevent the infection of diverse viruses by inhibiting viral protein expression (HSV-1, HCV, HPV, and CHIKV) and viral genome replication (HBV, HSV-1, and CHIKV). The 5' UTR activity of enteroviruses including poliovirus, EV71, CV-A16, and rhinovirus is crucial for viral RNA replication and protein translation (Gamarnik and Andino, 2000). In this study, we found that ADO did not affect the 5' UTR activity of EV-D68 in a viral RNA polymerase 3Ddependent $5^{\prime}$ UTR reporter assay, indicating that ADO targeted a step prior to the initiation of viral RNA and protein syntheses.

A previous study of the antiviral activity of ADO and its analogs showed that ADO prevented the entry of HSV-1 into cells (Aromdee et al., 2011). The life cycle of an enterovirus starts with binding of a cell surface receptor. Previous studies have demonstrated that sialic acid is required for the cellular entry of certain EV-D68 viruses (Liu et al., 2015a). To this end, our lab identified ICAM-5 as a functional receptor for both sialic acidsensitive and insensitive EV-D68 strains (Wei et al., 2016). In this study, we found that ADO did not influence the entry of EV-D68 into RD cells.

Enterovirus binds its specific receptor on the cell surface and then enters the host cell through receptor-mediated endocytosis. Subsequently, receptor binding or changes in $\mathrm{pH}$ in the virus-containing endosome trigger virus uncoating. Notably, EV-D68 is a unique respiratory enterovirus that is sensitive to acidic conditions and prefers lower culture temperatures (Oberste et al., 2004). Consistent with these properties, we found that acidification of the virus-containing endocytic vesicle was a critical step inhibited by ADO 
that ultimately inhibited EV-D68 infection. Consistent with this observation, previous studies have indicated that drugs inhibiting endosome acidification (Bafilomycin A1 and concanamycin A) prevented EV71 multiplication. Therefore, disrupting endosome acidification may represent an important antiviral strategy for therapeutic development against EV-D68. Furthermore, ADO has been shown to inhibit autophagosome-lysosome fusion and suspended autophagy processes (Zhou et al., 2012). Future studies are needed to identify the cellular targets of ADO that inhibit endosome/autophagosome maturation.

The work described herein provides compelling evidence that ADO inhibits EV-D68 replication by preventing endosome acidification as a novel antiviral property. Our results show virushost interactions and highlight the potential therapeutic utility of $\mathrm{ADO}$ as a clinical therapeutic against EV-D68 infection.

\section{AUTHOR CONTRIBUTIONS}

DyW, HG, JC, and DW performed the experiments. DyW, HG, PG, and WW analyzed the data. WW, DyW, PG, and BL wrote

\section{REFERENCES}

Aromdee, C., Suebsasana, S., Ekalaksananan, T., Pientong, C., and Thongchai, S. (2011). Stage of action of naturally occurring andrographolides and their semisynthetic analogues against herpes simplex virus type 1 in vitro. Planta Med. 77, 915-921. doi: 10.1055/s-0030-1250659

Baggen, J., Thibaut, H. J., Staring, J., Jae, L. T., Liu, Y., Guo, H., et al. (2016). Enterovirus D68 receptor requirements unveiled by haploid genetics. Proc. Natl. Acad. Sci. U.S.A. 113, 1399-1404. doi: 10.1073/pnas.1524498113

Baggen, J., Thibaut, H. J., Strating, J. R. P. M., and Van Kuppeveld, F. J. M. (2018). The life cycle of non-polio enteroviruses and how to target it. Nat. Rev. Microbiol. 16, 368-381. doi: 10.1038/s41579-018-0005-4

Blomqvist, S., Savolainen, C., Raman, L., Roivainen, M., and Hovi, T. (2002). Human rhinovirus 87 and enterovirus 68 represent a unique serotype with rhinovirus and enterovirus features. J. Clin. Microbiol. 40, 4218-4223. doi: 10.1128/JCM.40.11.4218-4223.2002

Calabrese, C., Berman, S. H., Babish, J. G., Ma, X., Shinto, L., Dorr, M., et al. (2000). A phase I trial of andrographolide in HIV positive patients and normal volunteers. Phytother. Res. 14, 333-338. doi: 10.1002/1099-1573(200008)14: 5<333::AID-PTR584>3.0.CO;2-D

Chen, H., Ma, Y. B., Huang, X. Y., Geng, C. A., Zhao, Y., Wang, L. J., et al. (2014). Synthesis, structure-activity relationships and biological evaluation of dehydroandrographolide and andrographolide derivatives as novel antihepatitis B virus agents. Bioorg. Med. Chem. Lett. 24, 2353-2359. doi: 10.1016/j. bmcl.2014.03.060

Ding, Y., Chen, L., Wu, W., Yang, J., Yang, Z., and Liu, S. (2017). Andrographolide inhibits influenza A virus-induced inflammation in a murine model through NF-kappaB and JAK-STAT signaling pathway. Microbes Infect. 19, 605-615. doi: 10.1016/j.micinf.2017.08.009

Gamarnik, A. V., and Andino, R. (2000). Interactions of viral protein 3CD and poly $(\mathrm{rC})$ binding protein with the 5 ' untranslated region of the poliovirus genome. J. Virol. 74, 2219-2226. doi: 10.1128/JVI.74.5.2219-2226.2000

Greninger, A. L., Naccache, S. N., Messacar, K., Clayton, A., Yu, G., Somasekar, S., et al. (2015). A novel outbreak enterovirus D68 strain associated with acute flaccid myelitis cases in the USA (2012-14): a retrospective cohort study. Lancet Infect. Dis. 15, 671-682. doi: 10.1016/S1473-3099(15)70093-9

Huang, Y. P., Lin, T. L., Lin, T. H., and Wu, H. S. (2017). Molecular and epidemiological study of enterovirus D68 in Taiwan. J. Microbiol. Immunol. Infect. 50, 411-417. doi: 10.1016/j.jmii.2015.07.015

Ishiko, H., Miura, R., Shimada, Y., Hayashi, A., Nakajima, H., Yamazaki, S., et al. (2002). Human rhinovirus 87 identified as human enterovirus 68 by the paper with help from all authors. WW, PG, and BL directed the project.

\section{FUNDING}

This work was supported in part by funding from the National Natural Science Foundation of China (Grant Nos. 81772183, 31600132, and 31800150), the Chinese Ministry of Science and Technology (Grant No. 2018ZX10731101-001-016), the Department of Science and Technology of Jilin Province (Grant No. 20180101127JC), the Program for JLU Science and Technology Innovative Research Team (Grant No. 2017TD08), and the Fundamental Research Funds for the Central Universities.

\section{ACKNOWLEDGMENTS}

We thank Jiaxin Yang, Yuanyuan Li, Guoqiang Zhou, and Chuanyan Dai for technical assistance and Editage for editorial assistance.

VP4-based molecular diagnosis. Intervirology 45, 136-141. doi: 10.1159/0000 65866

Jayakumar, T., Hsieh, C. Y., Lee, J. J., and Sheu, J. R. (2013). Experimental and clinical pharmacology of andrographis paniculata and its major bioactive phytoconstituent andrographolide. Evid. Based Complement. Alternat. Med. 2013:846740. doi: 10.1155/2013/846740

Kaida, A., Kubo, H., Sekiguchi, J., Kohdera, U., Togawa, M., Shiomi, M., et al. (2011). Enterovirus 68 in children with acute respiratory tract infections. Osaka Japan. Emerg. Infect. Dis. 17, 1494-1497. doi: 10.3201/eid1708. 110028

Kumar, R. A., Sridevi, K., Kumar, N. V., Nanduri, S., and Rajagopal, S. (2004). Anticancer and immunostimulatory compounds from Andrographis paniculata. J. Ethnopharmacol. 92, 291-295. doi: 10.1016/j.jep.2004.03.004

Lagache, T., Danos, O., and Holcman, D. (2012). Modeling the step of endosomal escape during cell infection by a nonenveloped virus. Biophys. J. 102, 980-989. doi: 10.1016/j.bpj.2011.12.037

Lee, J. C., Tseng, C. K., Young, K. C., Sun, H. Y., Wang, S. W., Chen, W. C., et al. (2014). Andrographolide exerts anti-hepatitis $C$ virus activity by up-regulating haeme oxygenase-1 via the $\mathrm{p} 38$ MAPK/Nrf2 pathway in human hepatoma cells. Br. J. Pharmacol. 171, 237-252. doi: 10.1111/bph.12440

Liu, Y., Sheng, J., Baggen, J., Meng, G., Xiao, C., Thibaut, H. J., et al. (2015a). Sialic acid-dependent cell entry of human enterovirus D68. Nat. Commun. 6:8865. doi: $10.1038 /$ ncomms 9865

Liu, Y., Sheng, J., Fokine, A., Meng, G., Shin, W. H., Long, F., et al. (2015b). Structure and inhibition of EV-D68, a virus that causes respiratory illness in children. Science 347, 71-74. doi: 10.1126/science.1261962

Maloney, J. A., Mirsky, D. M., Messacar, K., Dominguez, S. R., Schreiner, T., and Stence, N. V. (2015). MRI findings in children with acute flaccid paralysis and cranial nerve dysfunction occurring during the 2014 enterovirus D68 outbreak. AJNR Am. J. Neuroradiol. 36, 245-250. doi: 10.3174/ajnr.A4188

Mertz, D., Alawfi, A., Pernica, J. M., Rutherford, C., Luinstra, K., and Smieja, M. (2015). Clinical severity of pediatric respiratory illness with enterovirus D68 compared with rhinovirus or other enterovirus genotypes. CMAJ 187, 12791284. doi: 10.1503/cmaj.150619

Messacar, K., Abzug, M. J., and Dominguez, S. R. (2015). 2014 outbreak of enterovirus D68 in North America. J. Med. Virol. 88, 739-745. doi: 10.1002/ jmv. 24410

Messacar, K., Hawkins, S. M., Baker, J., Pearce, K., Tong, S., Dominguez, S. R., et al. (2016). Resource burden during the 2014 enterovirus D68 respiratory disease outbreak at children's hospital colorado: an unexpected strain. JAMA Pediatr. 170, 294-297. doi: 10.1001/jamapediatrics.2015.3879 
Midgley, C. M., Jackson, M. A., Selvarangan, R., Turabelidze, G., Obringer, E., Johnson, D., et al. (2014). Severe respiratory illness associated with enterovirus D68 - Missouri and Illinois, 2014. MMWR Morb. Mortal. Wkly. Rep. 63, 798-799.

Midgley, C. M., Watson, J. T., Nix, W. A., Curns, A. T., Rogers, S. L., Brown, B. A., et al. (2015). Severe respiratory illness associated with a nationwide outbreak of enterovirus D68 in the USA (2014): a descriptive epidemiological investigation. Lancet Respir. Med. 3, 879-887. doi: 10.1016/S2213-2600(15)00335-5

Oberste, M. S., Maher, K., Schnurr, D., Flemister, M. R., Lovchik, J. C., Peters, H., et al. (2004). Enterovirus 68 is associated with respiratory illness and shares biological features with both the enteroviruses and the rhinoviruses. J. Gen. Virol. 85, 2577-2584. doi: 10.1099/vir.0.79925-0

Oermann, C. M., Schuster, J. E., Conners, G. P., Newland, J. G., Selvarangan, R., and Jackson, M. A. (2015). Enterovirus d68. A focused review and clinical highlights from the 2014 U.S. Outbreak. Ann. Am. Thorac. Soc. 12, 775-781. doi: 10.1513/AnnalsATS.201412-592FR

Panraksa, P., Ramphan, S., Khongwichit, S., and Smith, D. R. (2017). Activity of andrographolide against dengue virus. Antiviral Res. 139, 69-78. doi: 10.1016/j. antiviral.2016.12.014

Reed, L. J., and Muench, H. (1938). A simple method of estimating fifty per cent endpoints. Am. J. Hyg. 27, 493-497.

Reiche, J., Bottcher, S., Diedrich, S., Buchholz, U., Buda, S., Haas, W., et al. (2015). Low-level circulation of enterovirus D68-associated acute respiratory infections. Germany, 2014. Emerg. Infect. Dis. 21, 837-841. doi: 10.3201/ eid2105.141900

Rhoden, E., Zhang, M., Nix, W. A., and Oberste, M. S. (2015). In vitro efficacy of antiviral compounds against enterovirus D68. Antimicrob. Agents Chemother. 59, 7779-7781. doi: 10.1128/AAC.00766-15

Schieble, J. H., Fox, V. L., and Lennette, E. H. (1967). A probable new human picornavirus associated with respiratory diseases. Am. J. Epidemiol. 85, 297-310. doi: 10.1093/oxfordjournals.aje.a120693
Sun, L., Meijer, A., Froeyen, M., Zhang, L., Thibaut, H. J., Baggen, J., et al (2015). Antiviral activity of broad-spectrum and enterovirus-specific inhibitors against clinical isolates of enterovirus D68. Antimicrob. Agents Chemother. 59, 7782-7785. doi: 10.1128/AAC.01375-15

Wei, W., Guo, H., Chang, J., Yu, Y., Liu, G., Zhang, N., et al. (2016). ICAM-5/telencephalin is a functional entry receptor for enterovirus D68. Cell Host Microbe 20, 631-641. doi: 10.1016/j.chom.2016. 09.013

Wintachai, P., Kaur, P., Lee, R. C., Ramphan, S., Kuadkitkan, A., Wikan, N., et al. (2015). Activity of andrographolide against chikungunya virus infection. Sci. Rep. 5:14179. doi: 10.1038/srep14179

Yu, B., Dai, C. Q., Jiang, Z. Y., Li, E. Q., Chen, C., Wu, X. L., et al. (2014) Andrographolide as an anti-H1N1 drug and the mechanism related to retinoic acid-inducible gene-I-like receptors signaling pathway. Chin. J. Integr. Med. 20, 540-545. doi: 10.1007/s11655-014-1860-0

Zhou, J., Hu, S. E., Tan, S. H., Cao, R., Chen, Y., Xia, D., et al. (2012). Andrographolide sensitizes cisplatin-induced apoptosis via suppression of autophagosome-lysosome fusion in human cancer cells. Autophagy 8, 338-349. doi: $10.4161 /$ auto. 18721

Conflict of Interest Statement: The authors declare that the research was conducted in the absence of any commercial or financial relationships that could be construed as a potential conflict of interest.

Copyright (c) 2018 Wang, Guo, Chang, Wang, Liu, Gao and Wei. This is an openaccess article distributed under the terms of the Creative Commons Attribution License (CC BY). The use, distribution or reproduction in other forums is permitted, provided the original author(s) and the copyright owner(s) are credited and that the original publication in this journal is cited, in accordance with accepted academic practice. No use, distribution or reproduction is permitted which does not comply with these terms. 